GLUON PRODUCTION AMPLITUDES FOR $9 \bar{q}$ HIGH-ENERGY BACKWARD SCATTERING ${ }^{*}$ )

\title{
B.I. Ermolaev
}

A.F. Ioffe Physico-Technical Institute Ac ademy of Science of the USSR

194021 Leningrad, USSR

and

L.N. Lipatov

CERN - Geneva

and

Leningrad Institute of Nuclear Physics

Ac ademy of Science of the USSR

188350 Gatchina, USSR

\begin{abstract}
A factorization method is used for the investigation of gluon bremstrahlung in the quark-antiquark backward scattering. The differential equations for scattering amplitudes are obtained in a double-logarithmic approximation. These equations are solved for some cases of interest.
\end{abstract}

*) While this paper was finalized during L. Lipatov's visit to CERN and is circulated as a CERN preprint, it is here reproduced as it was typed in Leningrad. 


\section{Introduction}

Due to asymptotic freedom [1] it is possible to epply perturbative quantum chromodynamics (QCD) to the theoretical description of hadron reactions at small distances when both energy and momentum transfer are large enough. Here the parton-parton scattering appears as an elementarysub-process[2] Asymptotics of scattering amplitudes at high energy and fixed momentum transfer for the hard sub-processes can be calculated

in the leading-logarithmic approximation.

Inviramework of the complex angular momenta approach [3] the high energy asymptotics is determined by singularities of the cross channel partial waves. In the case of quark-antiquark forward scattering (the t-channel exchange of Pomeron pole with vacuum quantum numbers) the amplitudes were discussed in $\operatorname{Ref}$ [4] Similarly, the quark-antiquark backward scattering involves nonvacuum exchanges. The elastic amplitudes for this process were discussed in $\operatorname{Ref}[5]$.

Obviously, the backward quark-antiquark scattering is accompanied by considerable gluon bremsstrahlung of colliding objects. In a way, the situation is alike to $\mathrm{e}^{+} \mathrm{e}^{-}-$backward scattering that was examined in Ref [6] in the case when produced photons were emitted in certain multi-Regge kinematics. In the last reference $a$ Bethe-Salpeter-type equation for inelastic amplitudes was constructed. In principle ananalogous equation can be constructed for quark-antiquark backward scattering amplitudes also, but such approach would entail great technical difficulties.

Instead of that, we suggest a new factorization method to construct simplezequations for the gluon bremsstrah- 
lung amplitudes with external particles on their mass shell. The method is a generalization of the approach of Ref [5] to inelastic scattering. It has been applied recently to derive the inelastic form-factor for $\mathrm{e}^{+} \mathrm{e}^{-}$- annihilation into quarks and gluons [7]. The expression for it was guessed in Ref [8] (see also [9] ). Besides that, by using this method we have obtained the inelastic $e^{+} e^{--}$backward scattering amplitudes when emitted photons were in arbitrary multi-Regge kinematics [10].

Our object in this paper is to construct the differential equations for the quark-antiquark scattering amplitudes with the gluon bremsstrahlung taken into account. To a great extent, we will discuss multi-Regge kinematics. The explicit formulae for the amplitudes are given in some cases of interest.

Throughout the paper we use double-logarithmic approximation (DLA) but it is possible to generalize the method in order to take into account single logarithmic contributions.

In sec.2 we briefly review the factorization method in reference to an especially simple case of the elastic quark-antiquark backward scattering [5].

In Sec.3 the method is applied to the case of the single gluon emission in the $q \bar{q}$-backward scattering. We discuss also the cross. - channel colour structure of the production amplitude.

In Sec.4 we derive the differential equations for inelastic amplitudes for an arbitrary number of emitted gluons in the multiRegge kinematics. They take an especially simple form in terms of the $u$-channel partial waves. Solutions of these equations for some cases of interest are given. 
In Sec. 5 we briefly discuss non-multi-Regge kinematics of emitted gluons.

\section{The elastic $q \bar{q}$-backward scattering}

Let quark $Q i_{1}$ and antiquark $\bar{Q}_{i_{2}}$ convert after a hard collision into quark $Q^{\prime j_{2}}$ and antiquark $\bar{Q}_{j_{1}}^{\prime}$. Their momenta being $\mathrm{P}_{1}, \mathrm{P}_{2}$ for the initial particles and $\mathrm{P}_{1}^{\prime}, \mathrm{P}_{2}^{\prime}$ for the final particles correspondingly ( see Fig.1) . The indices $i_{1,2}, j_{1,2}$ label the colour states of the particles. We will discuss the Regge kinematical region for backward scattering

$$
|u| \ll s
$$

where

$$
S=\left(p_{1}+p_{2}\right)^{2}, \quad u=\left(p_{2}^{\prime}-p_{1}\right)^{2}
$$

Let us consider this process in the cross.. channel where quark states $\left|Q^{i_{1}} Q^{\prime j_{1}}\right\rangle$ and $\left|Q^{i_{2}} Q^{\prime j_{2}}\right\rangle$ play a part of initiel and final states correspondingly. It is convenient to expand the amplitude $M i_{i_{2}}^{i_{1}} \quad(s, u)$ into a sum of invariant amplitudes $\mathrm{M}_{\mathrm{T}}(\mathrm{s}, \mathrm{u})$ irreducible in colour

where

$$
M_{i_{2} j_{2}}^{i_{1} j_{1}}=\sum_{T=3,6} P_{T i_{2} j_{2}}^{i_{1} j_{1}} M_{T}
$$

$P_{T i_{2} j_{2}}^{i_{1} j_{1}}$ is the projecting operator into the irreducible $S U(3)$ - representation having dimension $T(T=3,6)$,

$$
P_{T i_{2} j_{2}}^{i_{1} j_{1}}=\frac{1}{2}\left(\delta_{i_{2}}^{i_{1}} \delta_{j_{2}}^{j_{1}}+\xi_{T} \delta_{j_{2}}^{i_{1}} \delta_{i_{2}}^{j_{1}}\right)
$$


and

$$
\begin{aligned}
& \xi_{T}=1 \text { corresponds to } T=6, \\
& \xi_{T}=-1 \text { corresponds to } T=3 .
\end{aligned}
$$

To calculate $\mathrm{H}_{\mathrm{T}}$ we have to take into account all appropriate Feynman diagrams. A number of such diagrams have an infrared divergence. To deal with this problem, we suggest a procedure for infrared regularization. We introduce an infrared cut-off $\mu$ for all virtual particles momenta $K_{i}^{\prime} \perp$ transversal to the plane containing $P_{1}$ and $P_{2}$ :

$$
\left|K_{i \perp}^{\prime}\right| \gg M
$$

where $K_{i}^{\prime}$ are momenta of virtual particles. Under condition (6) we can neglect masses of virtual particles and put them equal to zero. It was shown in Ref.[7] that, besides being infrared regulator, M takes place of a characteristic mass for invariant amplitudes (3). Therefore we write

$$
M_{T}=M_{T}\left(\frac{s}{\mu^{2}}, \frac{u}{\mu^{2}}\right)
$$

and define $U$-channel partial waves $f_{T}\left(\omega, u / \mu^{2}\right)$ by the Mellin transformation:

$$
M_{\mathrm{T}}=\int_{-i \infty}^{i \infty} \frac{d \omega}{2 \pi i}\left(\frac{s}{\mu^{2}}\right)^{\omega} f_{\mathrm{T}}\left(\omega, u / \mu^{2}\right) .
$$

Naturally, the value of $\mu$ must be large enough to apply the perturbative approach. 
As far as we use DLA, $K_{i \perp}^{\prime}$ satisfy one of the conditions ( $\left.\left|K_{i_{1} \perp}^{\prime}\right| \ll\left|K_{i_{2} \perp}^{\prime}\right| \ll \cdots\right)$. We denote $K_{\perp}^{\prime} \equiv K_{i_{1} \perp}^{\prime}$ the minimal transversal momentum of the virtual particles and name $i_{1}$ as the soft particle (though its energy may be large). Apparently, the integration region over $\left|K_{\perp}^{\prime}\right|$ may have $M$ as the lower limit unlike other loop transversal momenta. In turn, $\left|K_{\perp}^{\prime}\right|_{\text {is }}$ the lower limit for the integration over remaining $k_{i L}^{\prime}$.

The next step is to specify softparticles. There are two possibilities:

(i) The soffert particle is a gluon;

(ii) The sof particle is a quark. In this case the DLcontribution is possible only if there is another soft quark. (Fig.3). Thereby the amplitude factories into two pieces, every one being the same amplitude but with $M$ replaced by $\left|K_{\perp}^{\prime}\right|$, while $S$ is replaced by $2 \mathrm{P}_{1} \mathrm{~K}^{\prime}$ for the left blob and by $2 \mathrm{P}_{2} \mathrm{~K}^{\prime}$ for the right one. Both blobs are on-shell.

As for the integration region over $\left|K_{\perp}^{\prime}\right|$ there is an essential difference between two above discussed situations. For situation (i) the region is

$$
\left|K_{\perp}^{12}\right| \gg \int^{2}
$$

while for situation (ii) it is

$$
\left|K_{\perp}^{\prime 2}\right| \gg \max \left\{\mu^{2},|u|\right\}
$$

We shall call by the infrared region the region with the infrared cut-off equal to $\mu$. Hereafter, soft quarks have 
$-6-$

DI - contributions in the infrared region only if

$$
|u| \leqslant \mu^{2}
$$

$(11)$

We could obtain an integral equation for $M_{T}$ by summing the Born term and both $M$ - dependent and independent terms from Figs.2, 3 . Instead of that, we prefer to construct a: simplezdifferentidal equation for $M_{T}$ which involves $\mu$-dependent terms only. The equation is

$$
\left.\frac{\partial}{\partial \mu} M_{T}=\frac{\partial}{\partial \mu}\left[M_{T}^{(g l u o n}\right)+M_{T}^{(q u a r k)}\right](12)
$$

where $\mathrm{M}_{\mathrm{T}}$ (gluon) corresponds to the graphs in Fig. 2 (soft gluon contribution) and $M_{T}$ (quark) corresponds to the graph in Fig.3. There are two different cases to discuss:

$$
\begin{aligned}
& |u| \leqslant \mu^{2}, \\
& |u| \gg m^{2} .
\end{aligned}
$$

$(13 a)$

We shall keep the notation $\mathrm{M}_{\mathrm{T}}$ for the case (13a) while the amplitude for the case (13b)is denoted by M $\tilde{M}_{T}$ Let $f_{T}$ and $\tilde{f}_{T}$ be the partial waves for cases (13a) and (13b) correspondingly. Then by combining (8) and (12) one can obtain the equations (cf.Ref[5])

$$
\begin{aligned}
& \frac{d}{d x} f_{T}(x)-x f_{T}(x)+\frac{4 \pi^{2} \lambda}{2+N \xi_{T}}-\frac{1}{8 \pi^{2} \lambda} f_{T}^{2}(x)=0, \\
& \left(\frac{\partial}{\partial x}+\frac{\partial}{\partial z}\right) \widetilde{f}_{T}(x, z)=(x+2 \gamma z) \widetilde{f}_{T}(x, z) .
\end{aligned}
$$


Here $g$ is the QCD coupling constant and $x=\lambda^{-1} \omega$,

$$
\begin{aligned}
& z=-\lambda \ln \left(|u| / \mu^{2}\right), \quad \gamma=1 / 2\left(N \xi_{T}+2\right), \quad N=3, \\
& \lambda=\left[g^{2}\left(N^{2}+N \xi_{T}-2\right) / 8 \pi^{2} N\right]^{1 / 2} .
\end{aligned}
$$

It isn't difficult to solve eq.(14a) (the details see in Ref.[5])

$$
\begin{gathered}
f_{T}=-8 \pi^{2} \lambda \frac{d}{d x} \ln (d(x)), \\
d(x) \equiv \frac{1}{\Gamma(-p)} \int_{0}^{\infty} d y y^{-(1+p)} \exp \left(-x y-y^{2} / 2\right)=D_{p}(x) \exp \left(x^{2} / 4\right), \\
p=-\gamma,
\end{gathered}
$$

where $D_{p}$ is the parabolic cylinder function [11]. As for eq. $(14 b)$, we can easily obtain its first integral in the form

$$
\tilde{f}(x, z)=\Phi_{T}(x-z) \exp \left(x^{2} / 2+\gamma z^{2}\right) .
$$

Here $\phi_{T}$ is a smooth function.i To define $\Phi_{T}$ one can use the boundary condition

$$
\left.\widetilde{M}_{T}\left(\frac{s}{\mu^{2}}, \frac{u}{\mu^{2}}\right)\right|_{|u| \sim \mu^{2}}=M_{T}\left(\frac{s}{\mu^{2}}\right)
$$

where $\mathbb{M}_{\mathrm{T}}\left(S / \mu^{2}\right)$. corresponds to the case (13a). It is given by (8), (16) and therefore we have

$$
\Phi_{T}=-8 \pi^{2} \lambda \frac{d^{\prime}(x-z)}{d(x-z)} \exp \left[-(x-z)^{2} / 2\right],
$$




\section{Emission of a single gluon.}

Let us discuss the quark-antiquark backward scattering provided that the gluon $G_{b}^{a}$ is emitted. The indices $a, b$ denote its colour. The giuon momentum is $K_{1}$ (See Fig.4). It is convenient to introduce momentum transfers $q_{1}, q_{2}$ :

$$
q_{1}=p_{1}-p_{2}^{\prime}, q_{2}=q_{1}-K_{1}=p_{1}^{\prime}-p_{2} \text {. }
$$

We shall discuss the multi-Regge kinematics

$$
\left|u_{1,2}\right| \ll s_{1}, s_{2} \ll s
$$

where

$$
\begin{aligned}
& u_{1}=q_{1}^{2}, \quad u_{2}=q_{2}^{2}, \\
& s_{1}=\left(p_{2}^{\prime}+k_{1}\right)^{2}, \quad s_{2}=\left(k_{1}+p_{1}^{\prime}\right)^{2} .
\end{aligned}
$$

Again let us consider the process in the cross -channel where both initial and final quark states are the same as ones in Sec.2. Then the amplitude $M^{i_{1} j_{1} a} i_{2} j_{2} b$ can be expanded into the sum of the invariant amplitudes $\frac{(1)}{T_{1}, T_{2}} 1$ ) by

$$
M_{i_{2} j_{2} b}^{i_{1} j_{1} a}=\sum_{T_{1}, T_{2}} P_{T_{1}, T_{2} i_{2} j_{2} B}^{i_{1} j_{1} a} M_{T_{1}, T_{2}}^{(1)}
$$

where projecting operators ${ }^{P}{ }_{T_{1}, T_{2} i_{2} j_{2} b} i_{1} j_{1} a$ take the form:

$$
P_{T_{1}, T_{2} i_{2} j_{2} b}^{i_{1} j_{1} a}=\frac{1}{4}\left(\delta_{b}^{i_{1}} \delta_{r}^{j_{1}}+\xi_{T_{1}} \delta_{r}^{i_{1}} \delta_{b}^{j_{1}}\right) \text {. }
$$

1) As it is known [6] in DLA the spin structure of inelastic amplitudes coincides with that in the Born approximation. So, in the following discussion we omit in the amplitudes these Born factors. 
$\cdot\left(\delta_{i 2}^{r} \delta_{j 2}^{a}+\xi_{T_{2}} \delta_{i_{2}}^{a} \delta_{j 2}^{r}\right)-\frac{1}{6} \delta_{T_{1} T_{2}} \delta_{b}^{a}\left(\delta_{i_{2}}^{i_{1}} \delta_{j 2}^{j_{1}}+\xi_{T_{1}} \delta_{j 2}^{i_{1}} \delta_{i_{2}}^{j_{1}}\right)$ (25)

Here $\mathrm{T}_{1}\left(\mathrm{~T}_{2}\right)=1$ if the initial (the final) diquark state belongs to the symmetrical colour SU (3) - representation, while

$\mathrm{T}_{1}\left(\mathrm{~T}_{2}\right)=-1$ corresponds to antisymmetrical one. In DLA, the amplitudes $M_{T_{1}}, T_{2}$. are functions of both $U_{1,2}$ and $S_{1,2}$.

It is useful to have in mind that

$$
S_{1} S_{2}=S\left|K_{11}^{2}\right|
$$

as far as we consern with the multi-Regge kinematics (22).

In the first place, we shall discuss the diagonal amplitudes $\mathrm{M}_{\mathrm{T}}$ for which $\mathrm{T}_{1}=\mathrm{T}_{2} \equiv \mathrm{T}$. . If $u_{1} \sim u_{2}$ the amplitudes $\mathrm{M}_{\mathrm{T}}$ coincide with the elastic ones. In DLA there remains for examination the region

$$
\left|u_{1}\right| \gg\left|u_{2}\right|
$$

and the reversal one:. In the inelastic scattering, the condition (6) for infrared regulator must be supplemented with the restriction

$$
\mu \ll\left|K_{1 \perp}\right|
$$

In inelastic scattering, the definition of the soft virtual particle is more complicated as compared to the elastic case ( see [7]). The definition consists of the following steps:

To begin with, for every virtual particle $i$ with the momentum $K_{i}^{\prime}$ one can define $K_{i \perp}^{\prime}(A, B)$ as the component transversal with respect to the plane formed by the momenta $A$ and $B(A \neq B)$. After this, one can define $K_{i t}^{\prime}$ by 


$$
\left|K_{i t}^{\prime}\right|=\min _{A, B}\left\{\left|K_{i \perp}^{\prime}(A, B)\right|\right\}
$$

where $A, B$ run over $P_{1}, p_{1}^{\prime}, P_{2}, p_{2}^{\prime}, K_{1}$.

Then one has to take into consideration all virtual particles to extract the minimal value of $\left|K_{i t}^{\prime}\right|$ which we label $\left|K_{i, t}^{\prime}\right| \equiv\left|K_{t}^{\prime}\right|$. At last, one must compare $\left|K_{t}^{\prime}\right|$ with $\left|K_{1 \perp}\right|$ ( $K_{1} \perp^{\text {is }}$ the emitted gluon momentum transversal to the plane formed by the momenta $P_{1}$ and $P_{2}$ ). We define the soft virtual particle as particle $i_{1}$ provided that

$$
\left|K_{t}^{\prime}\right| \ll\left|K_{1 \perp}\right|
$$

The soft virtual gluon emitters are the external particles both quarks and the gluon $\mathrm{G}_{a}^{b}$. The integration over other loop momenta gives on-shell $\mathbb{M}_{\mathrm{T}}^{(1)}$ in which is substituted by $\left|K_{t}^{\prime}\right|$. The soft virtual quark situation is similar to the one in Sec.2. One can use the factorization to yield a differential equation for $\mathbb{M}_{\mathrm{T}}^{(1)}$. Naturally, the equation takes especially simple form in the terms of the partial waves which can be introduced by the Mellin transformation

$$
\begin{aligned}
& M_{T}^{(1)}\left(\frac{s_{1}}{\mu^{2}}, \frac{s_{2}}{\mu^{2}}, \frac{u_{1}}{\mu^{2}}, \frac{u_{2}}{\mu^{2}}\right)=\int_{-i \infty}^{i \infty} \frac{d \omega_{1}}{2 \pi i} \frac{d \omega_{2}}{2 \pi i}\left(\frac{s_{1}}{\mu^{2}}\right)^{\omega_{1} /}\left(\frac{s_{2}}{\mu^{2}}\right)^{\omega_{2}} f_{T}^{(x)}\left(\omega_{1}, \omega_{2}, \frac{u_{1}}{\mu^{2}}, \frac{u_{2}}{\mu^{2}}\right) .(31) \\
& \text { In the case }\left|u_{2}\right| \gg \mu^{2} \text { this equation is } \\
& -\left(\omega_{1}+\omega_{2}+u_{1} \frac{\partial}{\partial u_{1}}+u_{2} \frac{\partial}{\partial u_{2}}\right) f_{T}^{(1)}=-\frac{g^{2}}{8 \pi^{2}} R_{T}\left[\ln \left(\frac{\left|u_{1}\right|}{\mu^{2}}\right)+g^{2}(32)\right. \\
& \left.+\ln \left(\frac{\left|u_{2}\right|}{\mu^{2}}\right)\right] f_{T}^{(1)}-\frac{g^{2}}{8 \pi^{2}} \frac{C_{V}}{2} \ln \left(\frac{\left|K_{11}^{2}\right|}{\mu^{2}}\right) f_{T}^{(1)}-\frac{g^{2}}{8 \pi^{2}} \cdot f_{T}^{(1)} \\
& 2 C_{T}\left(\frac{\partial}{\partial \omega_{1}}+\frac{\partial}{\partial \omega_{2}}+\ln \left(\frac{\left|K_{11}^{2}\right|}{\mu^{2}}\right)\right) f_{T}
\end{aligned}
$$


where

$$
\begin{gathered}
C_{V}=N, \quad C_{T}=\left(N^{2}-2+\xi_{T} N\right) / 2 N, \\
R_{T}=\frac{N^{2}-1}{2 N}-C_{T} .
\end{gathered}
$$

The left - (right-) hand part of equation (32) corresponds to the left- (right-) hand part of the equation in Fig.5.

Let us explain the meaning of the terms in eq. (32). We shall consider non-convariant quantities in the cms frame. The terms in the left-hand part in (32) (just the first one in Fig.5) correspond to the derivative of $M_{T}^{(1)}$ with respect to $\mu$. The terms in the right-hand part of eq.(32) (and es. in Fig.5) correspond to the $\mu$-dependent DI soft gluon contributions. There are several angular regions which make this contributions. The first term in the right hand part of eq (32) (the first square bracket in Fig.5) represents the kinematics in which the soft gluon is emitted into a narrow angular region close to one of external quark momentum directions. The next term in (32) corresponds to the emission of the soft virtual gluon into a similar region around the external gluon momentum. The last term in (32) corresponds to the coherent emission of the soft virtual gluon by the initial (final) diquark state. Then $C_{T}$ is the square of the colour diquarkcharge of the state T.

For the case

$$
\left|u_{2}\right| \leqslant \mu^{2}
$$

$u_{2}$-dependent terms in eq (32) are absent but the soft virtual quark term $Q_{T}^{(1)}$ must be added to the equation: 


$$
Q_{T}^{(1)}=\frac{1}{8 \pi^{2}} f_{T}^{(1)}\left(x_{1}, x_{2}, u_{1} / \mu^{2}\right) f_{T}\left(x_{2}\right)
$$

where $f_{T}$ is given by (17).

The solution of eq (32) is (cf. [6]):

$$
\begin{aligned}
& M_{T}^{(1)}=-8 \pi^{2} \lambda^{2} \int_{-i \infty}^{i \infty} \frac{d l_{1}}{2 \pi i} \frac{d l_{2}}{2 \pi i}\left(\frac{s_{1}}{\left|u_{1}\right|}\right)^{\lambda l_{1}}\left(\frac{s_{2}}{\left|u_{1}\right|}\right)^{\lambda l_{2}} \frac{1}{\left(l_{2}-l_{1}+z_{2}-z_{1}\right)} \cdot \\
& \cdot\left[\frac{d^{\prime}\left(l_{1}\right)}{d\left(l_{1}\right)}-\frac{d^{\prime}\left(l_{2}+z_{2}-z_{1}\right)}{d\left(l_{2}+z_{2}-z_{1}\right)}\right] \frac{d\left(l_{2}+z_{2}-z_{1}\right)}{d\left(l_{2}\right)} \cdot \\
& \cdot \exp \left\{\lambda z_{1} \ln \left(s_{1} /\left|u_{1}\right|\right)+\lambda z_{2} \ln \left(s_{2} /\left|u_{1}\right|\right)-z_{1}^{2} / 2+\gamma\left(z_{1}^{2}+z_{2}^{2}\right) / 2-\right. \\
& \left.-\left(g^{2} / 16 \pi^{2}\right)\left(C_{V} / 2\right) \ln \left(\left|k_{11}^{2}\right| / \mu^{2}\right)\right\} .
\end{aligned}
$$

The variables $l_{1,2}$ are related to the complex angular momenta $j_{1,2}$ by

$$
j_{i}=\lambda \ell_{i}=\omega_{i}-\lambda z_{i} \quad(i=1,2)
$$

while

$$
z_{1,2}=-\lambda \ln \left(\left|u_{1,2}\right| / \mu^{2}\right)
$$

To derive (36) from (32) we used the boundary conditions

$$
\begin{gathered}
\left.M_{T}^{(1)}\left(\frac{s_{1}}{\mu^{2}}, \frac{s_{2}}{\mu^{2}}, \frac{u_{1}}{\mu^{2}}, \frac{u_{2}}{\mu^{2}}\right)\right|_{\left|u_{2}\right| \sim \mu^{2}}=\widetilde{M}_{T}^{(1)}\left(\frac{s_{1}}{\mu^{2}}, \frac{s_{2}}{\mu^{2}}, \frac{u_{1}}{\mu^{2}}\right), \text { (39) } \\
\left.\widetilde{M}_{T}^{(1)}\left(\frac{s_{1}}{\mu^{2}}, \frac{s_{2}}{\mu^{2}}, \frac{u_{1}}{\mu^{2}}\right)\right|_{\left|u_{1}\right| \sim \mu^{2}}=M_{T}\left(\frac{s}{\mu^{2}}\right),
\end{gathered}
$$


where $\widetilde{M}_{T}^{(1)}\left(s_{1} / \mu^{2}, s_{2} / \mu^{2}, u_{1} / \mu^{2}\right)$ is the amplitude for the case (34) Let us return to the non-diagonal amplitudes $M_{T_{1}, T_{2}}^{(1)}$ for which $\mathrm{T}_{1} \neq \mathrm{T}_{2}$. One can show that they are absent on DLA: Indeed if $U_{1} \rightarrow U_{2}$ then $M_{T_{1}, T_{2}}^{(1)}$ transform themselves into the elastic amplitudes $\widetilde{M} T_{1}, T_{2}$ $=\delta_{T_{1}, T_{2}} \widetilde{M}_{T_{1}}$ (see Sec.2) becauge of the colour invariance.

\section{I gluons emitted.}

Let the total number of emitted gluons $b e n$, their momenta being $K_{1}, K_{2}, \ldots, K_{n}$ one can introduce $q_{i}$ (see Fig.6) to IabeI momenta of the intermediate crossing-channel states

$$
q_{1}=p_{1}-p_{2}^{\prime}, q_{2}=q_{1}-K_{1}, \ldots, \quad q_{n+1}=q_{n}-K_{n}=p_{1}^{\prime}-p_{2} \text {. }
$$

We fix multi-Regge kinematics:

$$
\begin{aligned}
& p_{1} q_{1} \ll p_{1} q_{2} \ll \ldots \ll p_{1} q_{n+1}, p_{2} q_{1} \gg p_{2} q_{2} \gg \cdots \gg p_{2} q_{n+1}, \\
& \left|q_{i_{1}}^{2}\right| \leqslant\left|q_{i_{2}}^{2}\right| \leqslant \ldots \leqslant\left|q_{i_{n+1}}^{2}\right| \quad\left(i_{1}, \ldots, i_{n+1}=1, \ldots, n+1\right) .
\end{aligned}
$$

As well as the case $n=1$ we can proceed to the invariant amplitudes $M_{T}^{(n)}, \ldots, T_{n+1}$. They are functions of $S_{i}$ and $u_{i}$ where

$$
\begin{aligned}
& s_{1}=\left(p_{2}^{\prime}+K_{1}\right)^{2}, s_{2}=\left(K_{1}+k_{2}\right)^{2}, \ldots, s_{n+1}=\left(K_{n}+p_{1}^{\prime}\right)^{2}, \\
& u_{1}=q_{1}^{2}, \quad u_{2}=q_{2}^{2}, \ldots, \quad u_{n+1}=q_{n+1}^{2} .
\end{aligned}
$$

It is not diffcult to generalize the soft virtual particle definitions to the present situation ( see $[7]$ ). We can define $\left|K_{i t}\right|$ as the minimal value of the gluon 1 momentum 
transversal to the plane formed by any pair of the external particle momenta $\left\{P_{1,2}, P_{1,2}^{\prime}, K_{1}, \ldots, K_{n}\right\}$ ( the pairs don't involve $\left.K_{i}\right)$. After this, one must select from them

$$
\left|K_{i_{1} t}\right|=\min \left\{\left|K_{i t}\right|\right\} \equiv\left|K_{t}\right| \text {. }
$$

By adding to the external momentum set in (29) the gluon momenta $K_{2}, \ldots, K_{n}$ it is possible to construct $\left|K_{t}^{\prime}\right|$ for the virtual particles.

Then $\mu$ is the infrared cut-off for these momenta:

$$
\mu \ll\left|K_{t}\right|,\left|K_{t}^{\prime}\right|
$$

and the virtual particle $i_{1}^{\prime}$ is the soft particle if

$$
\left|K_{i_{1}^{\prime} t}^{\prime}\right| \equiv\left|K_{t}^{\prime}\right| \ll\left|K_{t}\right|
$$

Also it is possible to pass to the invariant amplitudes $M_{T_{1}}^{(n)}, \ldots, T_{n+1}\left(\frac{s_{1}}{\mu^{2}}, \ldots, \frac{s_{n+1}}{\mu^{2}}, \frac{u_{1}}{\mu^{2}}, \ldots, \frac{u_{n+1}}{\mu^{2}}\right)$ and further to the partial waves $f_{T_{1}, \ldots, T_{n+1}}^{(n)}$ according to the Mellin transformation

$$
\begin{aligned}
& M_{T_{1}, \ldots, T_{n+1}}^{(n)}=\int_{-i \infty}^{i \infty} \frac{d \omega_{1}}{2 \pi i} \ldots \frac{d \omega_{n+1}}{2 \pi i}\left(\frac{s_{1}}{\mu^{2}}\right)^{\omega_{1}} \ldots\left(\frac{s_{n+1}}{\mu^{2}}\right)^{\omega_{n+1}} \\
& f_{T_{1}, \ldots, T_{n+1}}^{(n)}\left(\omega_{1}, \ldots, \omega_{n+1}, u_{1} / \mu^{2}, \ldots, u_{n+1} / \mu^{2}\right) .
\end{aligned}
$$

Again we shall discuss the diagonal amplitudes $M_{T}(n)$ in the first place. Let $\mathrm{T}_{1}=\mathrm{T}_{2} \cdot \cdot \mathrm{T}_{\mathrm{n}+1} \equiv \mathrm{T}$. Then the factorization of the soft particle makes it possible to sum DL contributions in the infrared region and to construct the equation for $f_{T}^{(n)}$. 
- $15-$

This equation is (cf.(32))

$$
\begin{aligned}
& -\left(\omega_{1}+\cdots+\omega_{n+1}+\sum_{i=1}^{n+1} u_{i} \frac{\partial}{\partial u_{i}}\right) f_{T}^{(n)}= \\
& -\frac{g^{2}}{8 \pi^{2}} R_{T}\left[\ln \left(\frac{\left|u_{1}\right|}{\mu^{2}}\right)+\ln \left(\frac{\left|u_{n+1}\right|}{\mu^{2}}\right)\right] f_{T}^{(n)}- \\
& -\frac{g^{2}}{8 \pi^{2}} 2 C_{T}\left[\frac{\partial}{\partial \omega_{1}}+\cdots+\frac{\partial}{\partial \omega_{n+1}}+\sum_{i=1}^{n} \ln \left(\frac{\left|K_{i 1}^{2}\right|}{\mu^{2}}\right)\right]_{T}^{(n)} \\
& -\frac{g^{2}}{8 \pi^{2}} \frac{C_{V}}{2}\left[\sum_{i=1}^{n} \ln \left(\frac{\left|K_{i 1}^{2}\right|}{\mu^{2}}\right)\right] f_{T}^{(n)}+Q_{T}^{(n)}
\end{aligned}
$$

The left-hand part of the equation (50) corresponds to the devivative of $M_{T}^{(n)}$ with respect to $\mu$. The terms in the right-hand part of (50) (except $Q_{T}^{(n)}$-term) correspond to the factorization of the soft gluon. The $Q_{T}^{(n)}$ is the contribution of the soft quarks. It appears if there are $u_{i_{k}}$ in (42), (44) for which $\left|u_{i_{k}}\right| \leqslant \mu^{2} \quad$ For example, if

$$
\left|u_{i_{1}}\right| \sim\left|u_{i_{2}}\right| \sim \ldots \sim\left|u_{i_{b}}\right| \sim \mu^{2}
$$

and

$$
i_{r_{1}} \neq i_{z_{2}} \pm 1
$$

for any $r_{1}, r_{2}<b$ 
then

$$
\begin{gathered}
Q_{T}^{(n)}=\frac{1}{8 \pi^{2}} \sum_{l=i_{1}}^{i_{b}} f_{T}^{(l-1)}\left(\omega_{1}, \ldots, \omega_{l}, u_{1} / \mu^{2}, \ldots, u_{l-1} / \mu^{2}\right) . \\
f_{T}^{(n+1-l)}\left(\omega_{l}, \ldots, \omega_{n+1}, u_{l+1} / \mu^{2}, \ldots, u_{n+1} / \mu^{2}\right) .
\end{gathered}
$$

Now we proceed to the general case when $\mathrm{T}_{i}$ are not necessarily equal. It can be shown that in DLA and in the multi-Regge kinematics such amplitudes vanish. We give a sketch of the proof :

i) One may solve eq (50) by iteration in the coupling constant.

(II) In the Born approximation, the production amplitides in the multi-Regge kinematics are diagonal in $\mathrm{T}_{i}$.

(iii) The soft virtual particle contributions do not change colour of $\mathrm{M}_{T_{1}}, \cdots, T_{n+1}$ on the right of eq $(50)$.

So, the emission of giluons does not change the n-channel colour.

Eq (50) can be solved for any specific set of momenta $q_{i_{k}}^{2}$ in (42) if we impose appropriate boundary conditions on $M_{T^{*}}^{(n)}$ One can use the recursion conditions. The amplitude $M_{\mathrm{T}}^{(n)}$ resolves itself into $M_{\mathrm{T}}^{\mathrm{n}-1}$ or $M^{(n-2)}$ when the restriction (52) breakes ( see [10]). Thus it is possible to pass from $M_{T}^{(n)}$ to $M_{T}$ by increasing in turns number of $q^{2} i_{k} \quad$ in $(51)$. 
We present solutions of this equation for some particular cases. In the first place we consider the multi-Regge kinematics with strongly-oxdered transfer momenta for which (42) takes the form

$$
i_{r}=n+2-r \quad(r=1, \ldots, n+1) .
$$

The "ordered" amplitude $\widetilde{M}_{T}^{(n)}$ is given by

$$
\begin{gathered}
\widetilde{M}_{T}^{(n)}=-8 \pi^{2} \lambda^{2} \int \frac{d l_{1}}{2 \pi i} \ldots \frac{d \ell_{n+1}}{2 \pi i}\left(\frac{s_{1}}{\left|u_{1}\right|}\right)^{\lambda \ell_{1}} \frac{d^{\prime}\left(\ell_{1}\right)}{d\left(\ell_{1}\right)} . \\
\prod_{i=2}^{n+1}\left\{\frac{1}{\left(\ell_{i+1}-l_{i}+z_{i+1}-z_{i}\right)}\left(\frac{s_{i}}{\left|u_{i-1}\right|}\right)^{\lambda \ell_{i}} \frac{d\left(l_{i}+z_{i}-z_{i-1}\right)}{d\left(\ell_{i}\right)}\right\} \cdot F_{n} \\
\cdot \exp \left[\lambda z_{1} \ln \left(\frac{s_{1}}{\left|u_{1}\right|}\right)+\sum_{i=2}^{n+1} \lambda z_{i} \ln \left(\frac{s i}{\left|u_{i-1}\right|}\right)-\frac{z_{1}^{2}}{2}+\frac{\gamma}{2}\left(z_{1}^{2}+z_{n+1}\right)\right] \\
F_{n}=\prod_{i=1}^{n} \exp \left\{-\frac{g^{2}}{16 \pi^{2}} \frac{C_{v}}{2} \ln ^{2}\left(\frac{\left|K_{i-1}^{2}\right|}{\mu^{2}}\right)\right\} .
\end{gathered}
$$

The expression (55) implies the following restrictions on integration contour positions

$$
R_{e}\left(l_{1}+z_{1}\right)<R_{e}\left(\ell_{2}+z_{2}\right)<\cdots<R_{e}\left(\ell_{n+1}+z_{n+1}\right) .
$$

The variables $I_{i}$ in (55) are related to the complex angular momenta $j_{i}$ by the formula

$$
j_{i}=\lambda l_{i}=\omega_{i}-\lambda z_{i} \quad(i=1, \ldots, n+1)
$$

while

$$
z_{i}=-\lambda \ln \left(\left|u_{i}\right| / \mu^{2}\right) \quad(i=1, \ldots, n+1) .
$$


Notations (58), (59) aze used for the other cases considered below.

Let us dwell upon $v^{\text {the }}=2$ case. Besides the "ordered" kinematics, there are two following independent multi-Regge kinematics:

$$
\mu^{2} \ll\left|u_{1}\right|,\left|u_{3}\right| \ll\left|u_{2}\right|
$$

and

$$
\mu^{2} \ll\left|u_{2}\right| \ll\left|u_{3}\right| \ll\left|u_{1}\right| .
$$

In the case $(60)$ the amplitude $M_{T}^{(2)}$ is given by

$$
\begin{aligned}
& M_{T}^{(2)}=8 \pi^{2} \lambda^{2} \int \frac{d l_{1}}{2 \pi i} \frac{d l_{2}}{2 \pi i} \frac{d l_{3}}{2 \pi i}\left(\frac{s_{1}}{\left|u_{2}\right|}\right)^{\lambda l_{1}}\left(\frac{s_{2}}{\left|u_{2}\right|}\right)^{\lambda l_{2}} . \\
& \left(\frac{s_{3}}{\left|u_{2}\right|}\right)^{\lambda l_{3}} \frac{1}{\left(l_{3}-\ell_{2}+z_{3}-z_{2}\right)} \frac{1}{\left(l_{2}-l_{1}+z_{2}-z_{1}\right)} \frac{d\left(l_{1}+z_{1}-z_{2}\right)}{d\left(l_{1}\right)} . \\
& \frac{d\left(l_{3}+z_{3}-z_{2}\right)}{d\left(l_{3}\right)} \frac{d^{\prime}\left(l_{2}\right)}{d\left(l_{2}\right)} \cdot F_{2} \cdot \exp \left[\lambda \sum_{i=1}^{3} z_{i} \ln \left(\frac{s i}{\left|u_{2}\right|}\right)-\frac{z_{2}^{2}}{2}+\right. \\
& \left.+\gamma\left(z_{1}^{2}+z_{3}^{2}\right) / 2\right] .
\end{aligned}
$$

It is assumed in (62) that the following disposition of contours in $I_{i}$ - planes takes place.

$$
\operatorname{Re}\left(l_{2}+z_{2}\right)<\operatorname{Re}\left(l_{1}+z_{1}\right)<\operatorname{Re}\left(l_{3}+z_{3}\right) \text {. }
$$

In the case (61) the corresponding expression for the amplitude $\widetilde{M}_{\mathrm{T}}^{(2)}$ is more complicated

$$
\widetilde{M}_{T}^{(2)}=M^{\prime}+M^{\prime \prime}
$$

where 


$$
\begin{aligned}
& M^{\prime}=-8 \pi^{2} \lambda^{2} \int \frac{d l_{1}}{2 \pi i} \frac{d l_{3}}{2 \pi i}\left(\frac{s_{1}}{\left|u_{1}\right|}\right)^{\lambda l_{1}}\left(\frac{s}{s_{1}}\right)^{\lambda l_{3}} \\
& \frac{1}{\left(l_{3}-l_{1}+z_{1}-z_{2}\right)} \frac{d^{\prime}\left(l_{1}\right)}{d\left(l_{1}\right)} \frac{d\left(l_{3}+z_{3}-z_{2}\right)}{d\left(l_{3}\right)} \cdot F_{2} \text {. } \\
& \cdot \exp \left[\lambda z_{1} \ln \left(\frac{s_{1}}{\left|u_{1}\right|}\right)+\lambda z_{3} \ln \left(\frac{s}{s_{1}}\right)-\frac{z_{1}^{2}}{2}+\frac{\gamma}{2}\left(z_{1}^{2}+z_{3}^{2}\right)\right] \text {, } \\
& M^{\prime \prime}=8 \pi^{2} \lambda^{2} \int \frac{d l_{1}}{2 \pi i} \frac{d l_{2}}{2 \pi i} \frac{d l_{3}}{2 \pi i}\left(\frac{s_{1}}{\left|u_{1}\right|}\right)^{\lambda l_{1}}\left(\frac{s_{2}\left|u_{2}\right|}{\left|u_{1} \cdot u_{3}\right|}\right)^{\lambda l_{2}} \text {. } \\
& \cdot\left(\frac{s_{3}}{\left|u_{3}\right|}\right)^{\lambda l_{3}} \frac{1}{\left(l_{3}-l_{2}+z_{3}-z_{2}\right)} \frac{1}{\left(l_{2}-l_{1}+z_{2}-z_{1}\right)} \frac{d^{\prime}\left(l_{1}\right)}{d\left(l_{1}\right)} . \\
& \frac{d^{\prime}\left(\ell_{3}\right)}{d\left(\ell_{3}\right)} d\left(\ell_{2}+z_{2}-z_{1}\right) d\left(\ell_{2}+z_{2}-z_{3}\right) \cdot \exp \left(\ell_{2}^{2} / 2\right) \text {. } \\
& \cdot\left[J\left(l_{2}\right)-J\left(l_{2}+z_{2}-z_{3}\right)\right] \cdot F_{2} \cdot \exp \left[z_{2}\left(z_{1}+z_{3}\right)+\right. \\
& \left.+\frac{\left(z_{1}^{2}+z_{3}^{2}-z_{2}^{2}\right)}{2}+\frac{\gamma\left(z_{1}^{2}+z_{3}^{2}\right)}{2}+\sum_{i=1}^{3} \lambda z_{i} \ln \left(\frac{s_{i}}{\mu^{2}}\right)\right] .
\end{aligned}
$$

$J(x)$ in (66) is given by

$$
J(x)=\int_{-i \infty}^{x} \frac{d t}{D_{p}^{2}(t)}=\frac{\sqrt{\pi}}{\Gamma(-p)} \frac{D_{p}(-x)}{D_{p}(x)}
$$

where we have used the following relation for the parabolic cylinder functions $D_{p}$

$$
\frac{d}{d t}\left(D_{p}(-t)\right) D_{p}(t)-D_{p}(-t) \frac{d}{d t} D_{p}(t)=\frac{\Gamma(-p)}{\sqrt{\pi}} . \quad(67 \text { a })
$$


The disposition of $1_{i}$ - contours in (65), (66) is

$$
\operatorname{Re}\left(\ell_{1}+z_{1}\right)<\operatorname{Re}\left(\ell_{3}+z_{3}\right)<\operatorname{Re}\left(\ell_{2}+z_{2}\right) .
$$

It is not difficult to see, that the singularities of the partial waves in all above cases are the Regge-poles. They are in $d$-functions zeroes.

\section{Non-multi-Regge kinematics}

In spite of the fact that we discussed oniy the amplitudes with the multi-Regge kinematics of emitted gluons it turns out that the virtual non-multi-Regge-gluons have been taken into account. They give exponential contributions in the formulae for the scattering amplitudes as well as the multi-Regge virtual gluons. This point was discussed in Ref. [6]. As for non-multi-Regge kinematics for the emitted real gluons, there is an important case of the cascade giuon emission [8].

Let the multi-Regge kinematics conditions(41) involve $n-n_{1}$ emitted gluons while the gluon cascade involves $n_{1}$ other gluons. We shall discuss the case in which these $n_{1}$ gluons are in the cascade induced by the gluon $r$.

Let us assume that both energies $\omega_{i}$ and emission angles $\theta_{i, j}$ of these gluons are strongly ordered by

$$
\begin{aligned}
& \omega_{r} \gg \omega_{1} \gg \omega_{2} \gg \cdots \gg \omega_{n_{1}} \\
& \theta_{1,2} \gg \theta_{2,1} \gg \theta_{3,2} \gg \cdots \gg \theta_{n_{1}, n_{1}-1}
\end{aligned}
$$

where $\theta_{A, B}$ is the angle between the gluon momenta $\vec{k}_{A}$ and $\vec{K}_{B}$. The conditions (69) mean that the gluon 1 is emitted by gluon $r$, the gluon 2 is emitted by gluon 1 etc. It is 
possible to show that the colour structure of the amplitude coincides with the Born colour structure ( see [8]). The invariant amplitude $\mathbb{M}(n)$ is given by

$$
\begin{aligned}
M^{(n)} & =M_{T}^{\left(n-n_{1}\right)} \exp \left\{-\frac{g^{2}}{16 \pi^{2}} \frac{C_{V}}{2}\left[\ln ^{2}\left(\frac{\omega_{1}^{2} \theta_{1,2}^{2}}{\mu^{2}}\right)+\right.\right. \\
& \left.\left.+\sum_{i=2}^{n_{1}} \ln ^{2}\left(\omega_{i}^{2} \theta_{i, i-1}^{2} / \mu^{2}\right)\right]\right\}
\end{aligned}
$$

where $\mathbb{M}_{\mathfrak{T}}^{\left(n-n_{i}\right)}$ is the multi-Regge amplitude. Expression may be generalized to more complicated cascades (cf.Refs. $[7,8]$ ). Acknowledgements

We are thankful to A.P.Bukhostov, V.S.Fadin, V.A.Khoze, M.Krawczyk, E.A.Kuraev and M.M.Terekhov for useful discussions. 
$R E F R E N C E S$

1. D.I.Gross and F.Wilczek, Phys.Rev.Lett. 30 (1973), 1343;

H.D.Politzer, Phys.Rev.Lett. ${ }^{30}(1973) 1346$.

2. R.P.Feynman, Photon-hadron interactions. (Benjamin, 1972);

L.N.Iipatov, Sov.Journal of NucI.Phys. 20 (1974) 94;

G.Altarelli and G.Parisi, Nucl.Phys.B 26 (1977) 298.

3. P.D.B.Collins, An introduction to Regge theory and high energy physics (Cambrige, 1977).

4. VoS.Fadin, I.N.Lipatov, E.A.Kuraev, JEIP 71 (1976) 840; ibid. 72 (1977) 377;

I.N.Lipatov, SovoJournal of Nucl.Phys. 23 (1976) 642; ibid 14 (1976) 71.

5. R.Kirschner and I.N.Lipatov, JETP 83 (1982) 488.

6. L.N.Lipatov, Sov.Journal of Nucl.Phys. 6 (1967) 564; 14 (1971) 160; 14 (1971) 396.

7. B.I.Ermolaev, V.S.Fadin, L.N.Iipatov, Sov.Journal of Nucl. Phys. 45 (1987), 817.

8. B.I.Ermolaev, V.S.Fadin, JEIP Lett. 33 (1981) 285;

A.H.Mueller, Phys.Lett. 104 B (1981) 161:

V.S.Fadin, SovoJournal of Nucl.Phys。 37 (1983) 408.

9. Y.I.Dokshitzer, VoA。Khoze, A。HoMueller and SoI.Troyan, CU-NP-374 (1987); RevoMod.Phys. (1988) in print.

10. B.I.Ermolaev and I。NoIipatov, A。F。Ioffe PTI preprint 1167 (1987).

11. EoT.Whittacker, G.M.Watson, Course of modern analysis. (Cambrige, 1927). 


\section{FIGURE CAPTIONS}

Fig.1. Elastic $q \bar{q}$-backward scattering.

Fig.2. DL-soft gluon contribution. $\left|K_{\perp}^{\prime}\right|$ is the infrared cut-off of the on-shell scattering amplitudes. $K^{\prime}$ is the soft gluon momentum.

Fig.3. DL-soft quark contribution. $\left|K_{\perp}^{\prime}\right|$ is the cut-off for both amplitudes. $K^{\prime}$ is the soft quark momentum.

Fig.4. Single gluon emission in $q \bar{q}$-backward scattering

Fig.5. Equation for $\mathbb{M}_{T}^{(1)}$ in the case $\left|u_{1}\right|,\left|u_{2}\right| \gg \mu^{2}$. The symboles inside the blobs denote the cut-off for on-shell amplitudes.

Fig.6. The soft quark contribution in the equation for $M_{T}^{(1)}$ in the case $\left|U_{1}\right| \gg\left|U_{2}\right| \sim \mu^{2} .\left|K_{t}^{\prime}\right|$ is the cut-off for both on-shell amplitudes.

Fig.7. Emission of $n$ bremsstrahlung gluons in the multiRegge kinematics. 


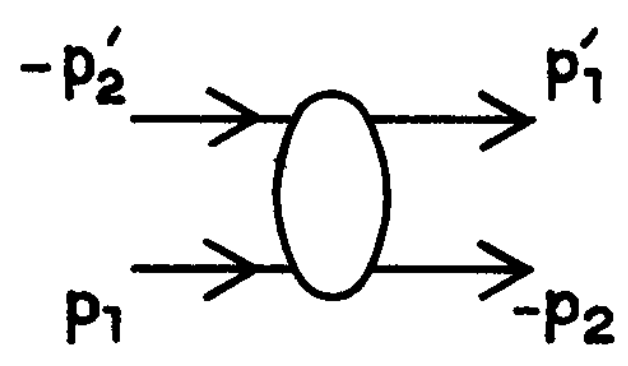

Fig. 7

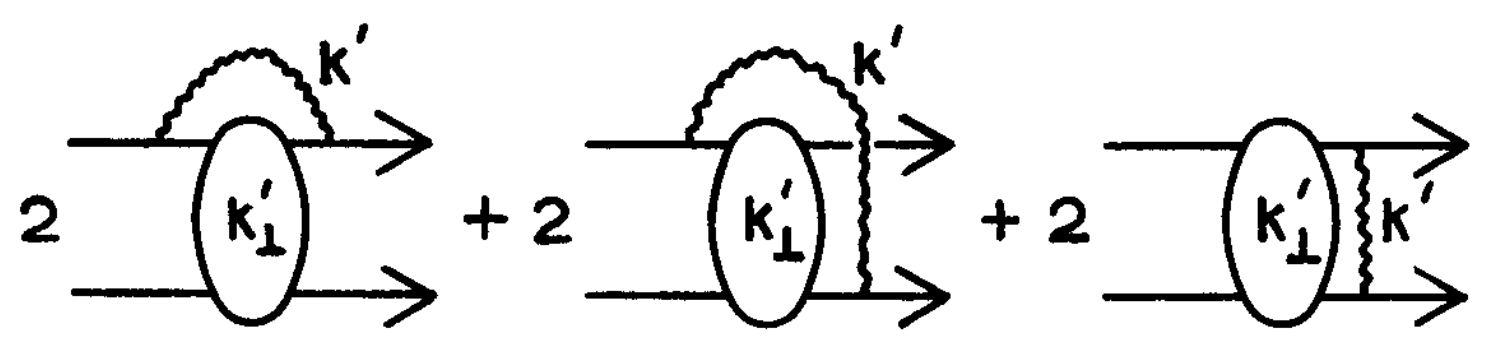

Fig. 2

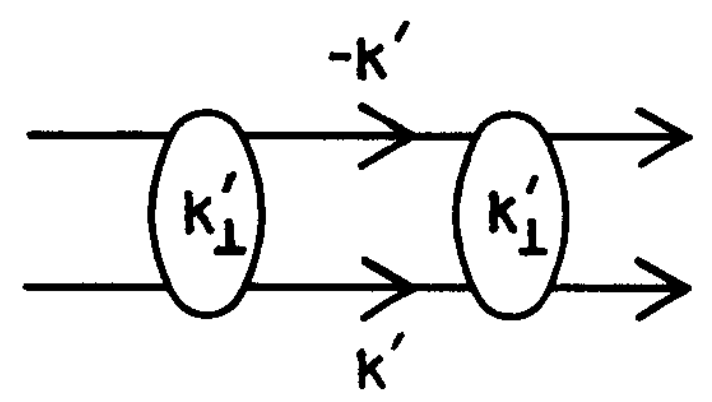

Fig. 3

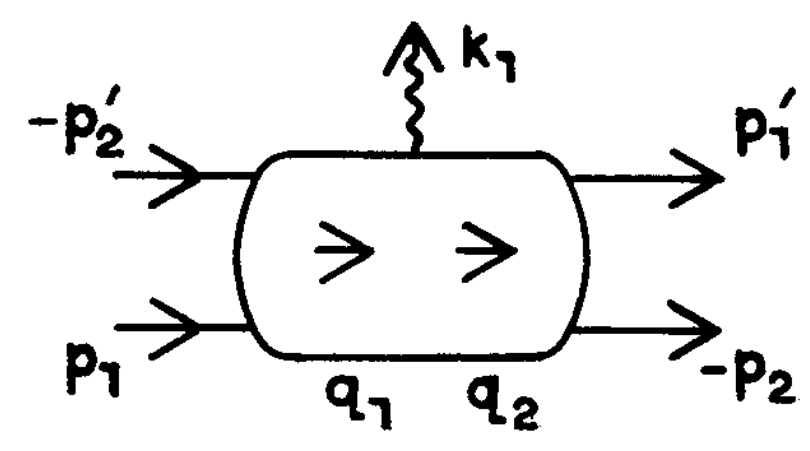

Fig. 4

B.I. Ermolaev, L. Ri. Lipator "Gluon production amplitudes.." 

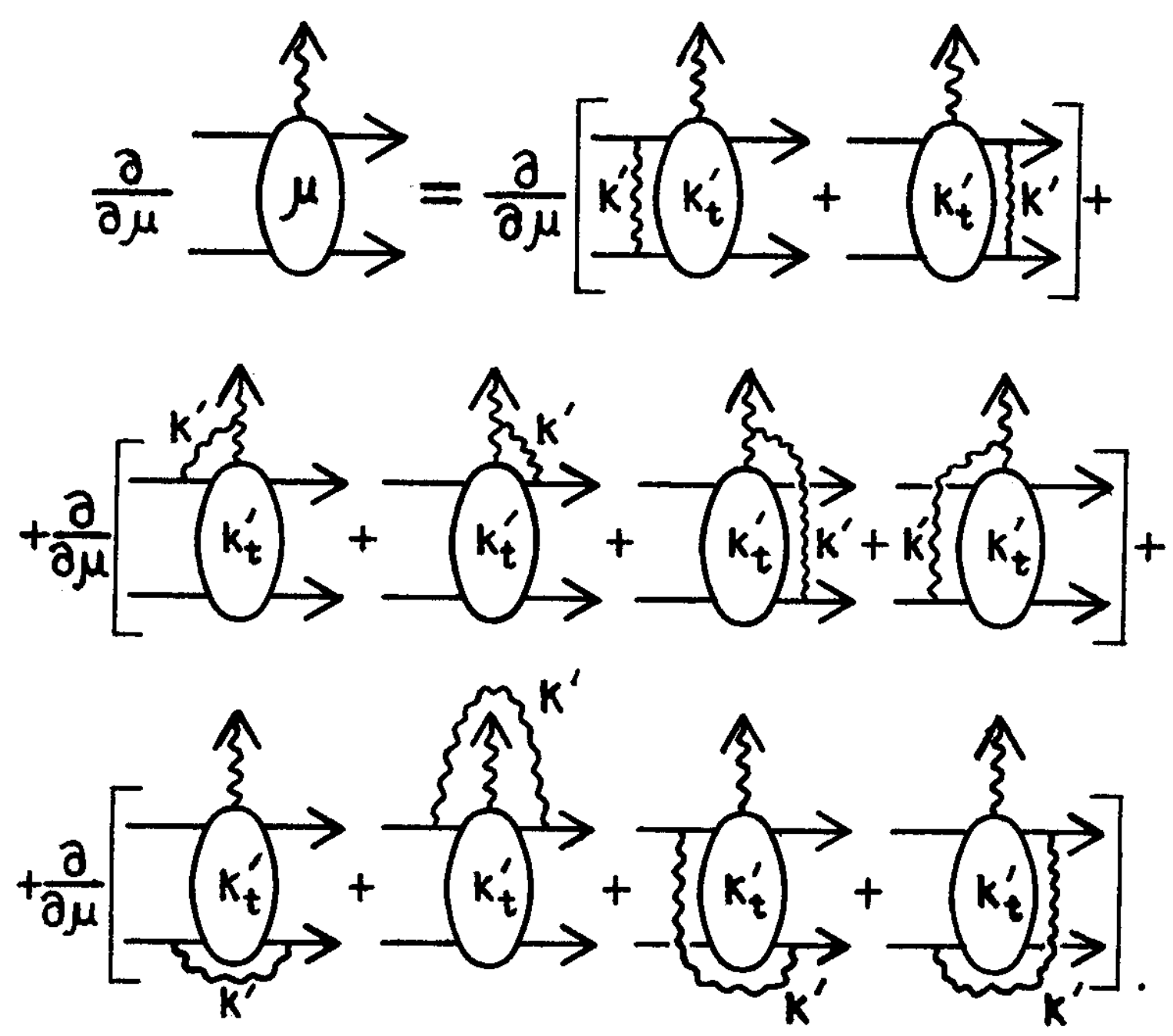

Fig. 5

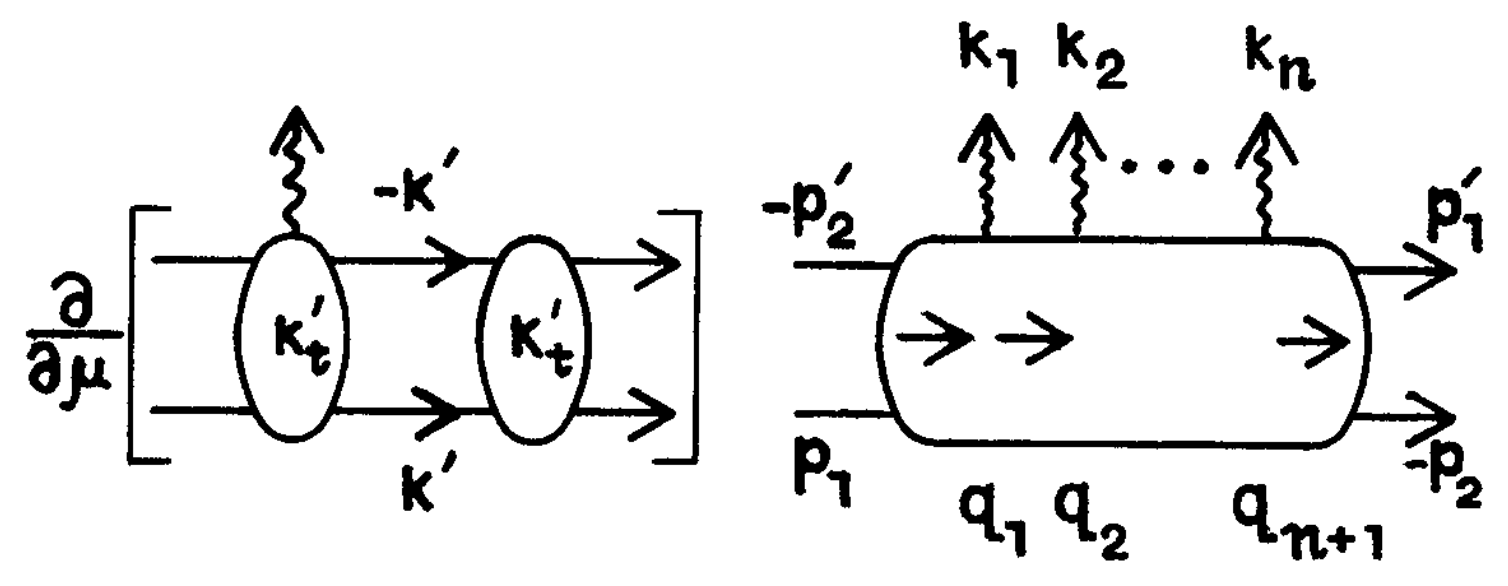

Fig. 6

Fig. 7 